\title{
Frontières
}

\section{Nous nous sommes rencontrées fin novembre 1997 au sud de la Chine...}

\section{Suzanne Lalonde}

Volume 14, numéro 1, automne 2001

Où est la marge ?

URI : https://id.erudit.org/iderudit/1074168ar

DOI : https://doi.org/10.7202/1074168ar

Aller au sommaire du numéro

Éditeur(s)

Université du Québec à Montréal

ISSN

1180-3479 (imprimé)

1916-0976 (numérique)

Découvrir la revue

Citer ce document

Lalonde, S. (2001). Nous nous sommes rencontrées fin novembre 1997 au sud de la Chine.... Frontières, 14(1), 72-74. https://doi.org/10.7202/1074168ar d'utilisation que vous pouvez consulter en ligne.

https://apropos.erudit.org/fr/usagers/politique-dutilisation/ 


\section{NOUS NOUS SOMMES RENCONTRÉES FIN NOVEMBRE 1997 AU SUD DE LA CHINE...}

\section{Suzanne Lalonde, psychologue ${ }^{1}$}

Nous nous sommes rencontrées fin novembre 1997 au sud de la Chine, à l'autre bout du monde. Elle s'est blottie tout doucement dans les bras que je lui tendais et elle y est demeurée de longues minutes, sans pleurer ni crier. Puis, j'ai senti le besoin de l'examiner un peu, ou ce que je pouvais en deviner au travers de nombreuses épaisseurs de vêtements. À quatorze mois, ma fille se tenait difficilement debout ou assise et les changements de couche étaient compliqués par une incapacité surprenante de ses petites jambes à s'entrouvrir pour faire place à une couche. Je m'étais toutefois bien préparée à la possibilité que mon enfant se présente de manière un peu inhabituelle, ce qui pouvait être attribuable à un manque important de stimulation et de soins à l'orphelinat. Cependant, on le comprendra, le raisonnement n'est pas à son meilleur dans des circonstances aussi magiques. Cette nouvelle aventure à deux généra une avalanche de sensations et de situations auxquelles chacune de nous tenta de s'adapter de son mieux. Le plaisir et l'attachement gagnèrent rapidement sur l'insécurité face à toutes ces expériences sans précédent.

Étrangement, dans la semaine qui a suivi, alors que mes compagnons magasinaient des vêtements et des chaussures pour

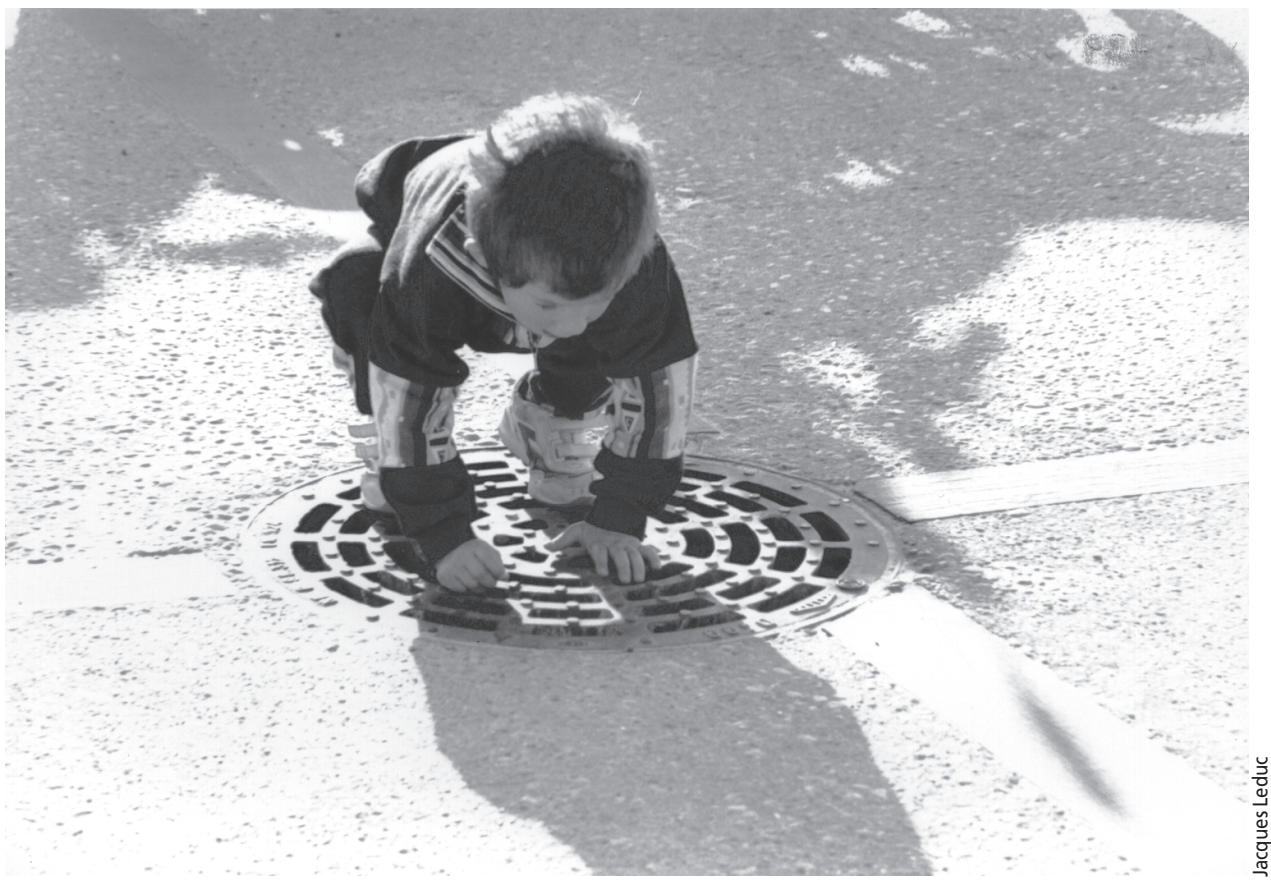

enfants, une voix intérieure émergeait de façon insistante pour me prier de ne pas acheter de souliers à Emma. Cela me parut un peu étrange, mais je décidai de ne pas en acheter pour le moment. Quelques jours à peine après notre retour de Chine, Emma fut diagnostiquée comme ayant une luxation bilatérale complète des deux hanches, une microcéphalie et d'importants retards de développement à divers niveaux. La vie en orphelinat laissa également ses traces: gale, exposition à la tuberculose, foie élargi à cause de la malnutrition, tête excessivement aplatie à l'arrière, un problème apparemment relié aux trop longues heures passées en position couchée. Rapidement sont apparus autour de nous deux des intervenants et des médecins spécialistes (seize 
champs de pratique différents ont été mis à contribution à ce jour) avec des demandes de consultation, des thérapies, des recommandations d'exercices à la maison ou encore des diagnostics. Un an plus tard, un examen des réflexes révéla également une atteinte neurologique, ce qui venait compromettre la qualité des résultats pouvant être obtenus par le biais des deux chirurgies à venir au niveau des hanches. La nature de cette atteinte neurologique ne fut précisée que quelque huit mois après ces chirurgies, sous la forme d'un déficit moteur cérébral.

Un lien important s'est très naturellement établi entre nous dès le début et je n'ai jamais remis en cause l'adoption de ma fille en rapport avec sa condition. Cependant, cette nouvelle réalité, avec toutes ses ramifications, me bouleversa profondément. Pendant les deux premières années (deux chirurgies majeures d'une durée totale de quinze heures prirent place au début de la deuxième année), mon humeur fut excessivement instable, alternant entre la colère et les pleurs intenses. Tout me paraissait infiniment lourd. Ma perception du temps se transforma alors que tous les aspects de son développement semblaient évoluer au compte-gouttes. Ma perception du futur se modifia radicalement, car je semblais ne pouvoir gérer que l'immédiat ou presque. Après chacune des deux chirurgies, Emma se retrouva pour une période totale de cinq mois dans un plâtre, des pieds au thorax. Puis d'autres formes d'immobilisation du bassin ont suivi. L'année suivante ses jambes affichèrent une rigidité inimaginable. Le langage de ma fille, qui était demeuré jusqu'alors très peu élaboré, disparut presque complètement. Tout était astreignant: tenter de la divertir, la laver, la nourrir, mes sorties pour acheter le nécessaire en veillant à ce que les réserves en question tiennent le coup jusqu'à la prochaine visite de la gardienne ou de l'auxiliaire familiale qui nous assista temporairement. Nos déplacements occasionnels pour revoir quelques spécialistes alors qu'Emma était toujours dans le plâtre se révélèrent une véritable épreuve : comment en hiver l'habiller adéquatement, la descendre d'un deuxième étage sans glisser et l'installer dans la voiture à l'aide de courroies adaptées fournies par l'hôpital?

Quelque temps avant l'adoption, j'avais contacté différents organismes afin de me renseigner sur le risque qui pouvait exister d'adopter sans le savoir un enfant présentant des problèmes de santé importants. Je n'oublierai jamais la voix irritée de ce parent adoptant, à l'autre bout du fil, qui me répondit: "Madame, vous devrez vous abandonner à cet enfant de la même manière que cet enfant s'abandonnera à vous. » Ce fil de sagesse me revient souvent à l'esprit et me soutient dans cette aventure avec ma fille. Ce parcours, avec ses défis, ses difficultés, et ses moments inoubliables, heureux et malheureux, a graduellement fait surgir en moi la volonté de trouver un sens à cette destinée. Cette longue et tortueuse réflexion s'est avérée très libératrice avec le temps. Je crois fermement que l'enfant que l'on reçoit (naturellement ou par le biais d'une adoption) est choisi par une instance supérieure, quelle qu'elle soit, et vise à nous faire progresser au plan spirituel. Je pense sincèrement que la présence d'Emma dans ma vie vise à faire de moi une meilleure personne et que cet accompagnement que je lui offre a également un sens face à son propre cheminement. Je prends conscience qu'intérieurement, dans les années qui ont précédé, je ressentais un vide important dans ma vie. J'entretenais le désir de pouvoir contribuer de manière significative et tangible à une cause sur le plan humain. Avec le recul, il m'apparaît extrêmement sécurisant et réconfortant de constater que ce que nous sommes, au plus profond de nous-même, est vu et entendu, et qu'un soutien est mis en place pour permettre à l'individu de s'actualiser à ce niveau. Je me suis également souvent prise à penser que si une enfant d'une telle beauté à bien des égards me fut destinée, et ce parmi des milliards d'individus, bien des choses paraissant jusque-là impossibles ne le sont plus.

Ce recul tellement essentiel face à ma réalité n'a malheureusement été possible que de manière limitée tout au long de ce périple. Quand on n'a pas un instant de répit, on s'épuise et cela contribue à prolonger le deuil et l'affect dépressif. Le parent pourra plus pleinement apprécier la réalité de même que les défis de son enfant handicapé et y être empathique dans la mesure où sa propre réalité n'est pas trop souffrante. Bien qu'il semble y avoir une volonté au plan des services sociaux à reconnaître le vécu des parents d'enfants handicapés et leurs besoins à cet égard, les fonds demeurent le plus souvent insuffisants pour affirmer adéquatement une telle volonté.

La vie d'un parent d'enfant handicapé est faite de contraintes multiples à différents niveaux: les thérapies et consultations médicales s'échelonnant sur plusieurs années, la dépendance prolongée de l'enfant au parent (pour se nourrir, marcher, parler, devenir propre), la nécessité fréquente de chirurgies en bas âge, la lenteur avec laquelle l'enfant parvient à exécuter le peu qu'il réussit à faire, la surcharge de poids dans la mesure où l'enfant ne marche que tardivement (s'il marche), les équipements orthopédiques à repositionner sur l'enfant selon qu'il est en période de repos, qu'on doit effectuer un changement de couche ou parce qu'il doit aller à la toilette, les poussettes adaptées et les chaises roulantes si lourdes à manier. Mentionnons également les nombreux échanges d'informations et les contacts avec les organismes et les prestataires de soins, les revendications à faire longtemps à l'avance pour assurer à notre enfant une place en garderie ou en service de garde au même titre que n'importe quel enfant. C'est alors que l'on constate l'existence des barrières, préjugés et anxiétés de la population face à l'intégration d'un enfant handicapé.

Même si elles semblent cocasses, certaines situations doivent être vécues pour que l'on puisse pleinement apprécier les frustrations qu'elles peuvent susciter. Ainsi, la petite n'est capable de faire seule quatre ou cinq pas que depuis juin 2000. Il me fallait donc, notamment l'hiver, l'installer dans le petit siège du chariot à provisions que l'on utilise pour faire l'épicerie. Toutefois, à cause de ses orthèses qui nécessitaient des bottes bien plus grandes pour ses pieds, il devenait extrêmement difficile d'entrer ses pieds dans les deux espaces prévus à cette fin tout en la soutenant dans les airs. Le tout prenait une allure encore plus pathétique alors que les bottes tombaient systématiquement au moment de la retirer du panier $\left(a ̀-20^{\circ} \mathrm{C}\right.$ comme ce fut souvent le cas). Et aussi, combien de fois me suis-je demandée le nombre de kilos que peut supporter une table à langer fixée au mur d'une salle de toilettes publiques alors que ma fille, encore aux couches, semblait nettement excéder le poids prévu par les fabricants. L'humour devient indispensable en de telles circonstances.

Plusieurs deuils ont été à faire à différents niveaux: perte de travail, tout d'abord, alors qu'il nous a fallu nous rapprocher d'un centre urbain à cause des soins de santé requis, perte importante de revenus due à la nécessité de soins prolongés après les chirurgies et à l'impossibilité croissante d'occuper un emploi à temps plein compte tenu des multiples obligations. Il devint également difficile, tout au moins pendant un certain temps, de fournir le même rendement à cause de la fatigue accrue, des priorités qui changent. J'ai déjà mentionné la perte d'équilibre au plan psychologique, perte du sens de l'humour alors que le stress prend le dessus et perte de la place occupée jusqu'alors au sein de la famille à mesure que les interactions se transforment de manière significative. Enfin, l'absence cruelle de temps libre a eu des répercussions sur ma condition physique, ma santé et mon univers affectif et sentimental. Comment parler par ailleurs du deuil à faire de l'enfant «normal », c'est-à-dire de ce désir d'un enfant qui ne requiert pas de 
soins prolongés en réadaptation ou de soins de santé particuliers. Cette réalité est doublement déchirante (et réconfortante à la fois) dans la mesure où cet enfant possède un caractère et des qualités tout à fait exceptionnels. C'est ainsi que je découvre chaque jour la détermination peu commune d'Emma, son intelligence vive, l'intensité avec laquelle elle vit chaque moment, sa grande beauté et sa sociabilité que je ne peux que lui envier, son humour, sa capacité impressionnante d'adaptation, toute son affection. Certes, il m'arrive de regarder avec envie les enfants des autres déambuler, marcher, courir, sauter avec une aisance toute naturelle. Par ailleurs, Emma, qui jusqu'à tout récemment faisait preuve d'un équilibre assez précaire en station debout, est toujours parvenue de manière tout à fait spontanée, à faire avec ses blocs des constructions qui défient presque les lois de la gravité. Je suis sûre que j'arriverais difficilement à faire tenir en équilibre ces mêmes structures, mais elle semble avoir une compréhension innée de ce qui favorise l'équilibre. Il m'arrive toujours de ressentir de la colère face à ses limites, mais je m'efforce plus que jamais de l'accueillir telle qu'elle est physiquement et mentalement, sans en attendre plus.

Comme c'est bien souvent le cas en situation de deuil prolongé, l'entourage (familial, médical, social, professionnel) prend rapidement ses distances face au vécu de la personne en cause. Une telle chose paraît liée à différents facteurs : l'ambiguïté initiale quant à la condition de l'enfant et le délai pour préciser les diagnostics qui maintiennent la personnesoutien en position de déséquilibre, l'ignorance des difficultés qu'une telle situation de vie peut engendrer, ou encore les possibilités limitées de répit qui nuisent à la capacité d'adaptation. À l'occasion, certains intervenants du domaine de la santé ou de la réadaptation tendent à minimiser notre vécu. Ce réflexe semble lié à l'impuissance et à un désir de se protéger contre la souffrance dont ils sont régulièrement les témoins puisqu'ils rencontrent fréquemment des personnes assez gravement handicapées. Comme le parent qui, à un certain niveau, est seul à porter le fardeau de cette réalité en ce qui a trait à son enfant parce qu'il connaît le plus intimement la façon avec laquelle tout cela se manifeste au quotidien, l'enfant lui aussi, à son propre niveau, porte seul la réalité de son corps, la seule qu'il connaisse. La difficulté pour Emma de se maintenir en équilibre, son désir de bouger, sa difficulté à demeurer attentive ou encore sa raideur musculaire influencent à chaque instant sa manière d'être au monde, et ce d'une manière que je ne peux qu'imaginer.
Quelques mots sur l'univers de la réadaptation qui est devenu partie intégrante de notre vie... On y entre sans jamais savoir quand on pourra en sortir, mais, en même temps, il est heureux de penser aux nombreuses améliorations possibles. Je me sens toujours réconfortée lorsque je pénètre dans ces lieux; j'ai le sentiment de me retrouver entourée d'individus qui vivent quelque chose de semblable à ce que nous vivons ou qui y sont réceptifs. Cette réadaptation qui, dans le cas de ma fille, vise à maximiser son potentiel au niveau du développement moteur et neurologique, du langage, de sa capacité à être attentive à ce qui se passe autour d'elle, fait une énorme différence quant à sa qualité de vie et à la mienne. Emma se sent de plus en plus en mesure de faire les choses comme les autres enfants. Elle est infiniment plus autonome, ce qui en retour lui donne de l'assurance. Le personnel qui nous entoure est chaleureux, disponible, attentif et compétent, et le contact avec mon enfant est positif. Ces personnes se manifestent dans notre vie sur une base individuelle, mais elles existent également à un niveau symbolique comme un ensemble, une extension à la famille en quelque sorte. Il arrive toutefois que des intervenants, de disciplines très diverses, sous-estiment l'importance de bâtir un lien positif à la fois avec l'enfant et le parent. Cela me paraît essentiel dans la mesure où une telle dyade (parent-enfant) évolue la plupart du temps dans un climat de très grande complicité et que le parent a tout à gagner d'un tel support au regard de ce qu'il cherche à accomplir quotidiennement avec son enfant.

Lorsque j'étais petite, mon père fréquentait régulièrement à une certaine époque un centre de réadaptation; il souffrait de problèmes d'arthrite, surtout aux mains. Il me plaisait toujours de l'accompagner et cela me permettait également de m'initier à différentes formes de thérapie. Je trouvais toutefois bouleversante la rencontre avec certaines de ces personnes et j'avais verbalisé mon inconfort à mon père. Celui-ci me répondit qu'il était important que je me familiarise avec une telle réalité. L'un des éléments difficiles auxquels je suis confrontée avec ma fille actuellement a trait à ce regard des autres qui nous envahit et qui nous suit une grande partie du temps à cause de sa démarche particulière et de l'appareillage qu'elle porte afin de rendre sa démarche plus fonctionnelle. Ce que je lis dans les yeux de ces gens varie passablement et se traduit difficilement en mots. J'ai conscience que trop souvent dans ma propre vie j'ai pu adopter l'un ou l'autre de ces regards. Je réalise avec le temps que bon nombre de ces façons d'aborder non verbalement la personne handicapée n'apporte aucun soutien aux personnes en cause. Je m'interroge quelquefois à savoir quel type de communication non verbale serait souhaitable et nourrissante dans un tel contexte. Ma fille entre maintenant dans un groupe d'âge où les enfants qui l'entourent sont de plus en plus aptes à verbaliser leur vécu et leurs préoccupations. Sa présence suscite donc très souvent nombre de questions en lien avec sa condition, questions qui sont tout à fait à propos compte tenu que sa situation n'a rien de familier. Pour le moment, ces questions qui laissent ma fille perplexe me font mal et mon impulsion est parfois de vouloir fuir la situation. Je ne gère pas toujours bien ces situations, mais suis très consciente que j'aurai un rôle crucial à jouer auprès d'elle quant au rapport qu'elle est appelée à entretenir avec sa condition et j'espère faciliter cet apprentissage. Je pense qu'il est très humain de ressentir de la tristesse face à une personne à qui il «manque » quelque chose, encore que cet aspect de manque soit bien discutable dans la mesure où nous sommes tous essentiellement des individus limités à un niveau ou à un autre (la différence étant que certains manques sont plus évidents ou moins bien acceptés socialement). L'intransigeance que l'on observe ici et là me rend inquiète par moments face à son futur. En même temps, j'aime à me rappeler la détermination, la force de caractère, le leadership et la sociabilité qui la caractérisent et qui la soutiendront tout au long de sa vie.

À ce jour, soit quatre ans plus tard, les professionnels qui nous entourent ne sont toujours pas en mesure de statuer exactement sur les limites d'Emma ou sur les aspects de son développement qui plafonneront éventuellement. Ainsi, nous ne savons toujours pas s'il lui sera possible d'intégrer le réseau scolaire régulier l'an prochain. Cette incertitude constante me donnait l'impression d'avoir également perdu une certaine capacité de définir l'avenir en quelque sorte. Avec le recul, il m'apparaît que cette assurance face à l'avenir n'est somme toute qu'une illusion que l'on se plaît à entretenir. J'ai toutefois la certitude qu'Emma n'a pas fini de m'étonner et moi, de grandir grâce à elle.

\section{Note}

1. Ce texte ne prétend en aucune manière représenter le vécu et la réalité des parents d'enfants handicapés. Il se veut essentiellement une occasion de se dire par un parent qui, à l'âge de 40 ans, a adopté seule une petite fille se révélant handicapée, et un récit de l'aventure qui en a découlé. L'auteure exerce la profession de psychologue, mais c'est avant tout à titre personnel qu'elle offre ce texte. Apparemment, une autre "adoption» se prépare dans cette petite famille, avec l'arrivée d'un conjoint. 